\title{
An Adaptive Cruise Control System Based on ARM CPU and PID for Ag- ricultural Vehicles
}

\author{
Li Xingshan ${ }^{1, *}$, Zhang Pingchuan ${ }^{2, *}$ and Zhu Jiayi ${ }^{2}$ \\ ${ }^{I}$ Medical Instrument Department, Luohe Medical College, Luohe, 462002, China \\ ${ }^{2}$ School of Information Engineering, Henan Institute of Science and Technology, Xinxiang, 453003, China
}

\begin{abstract}
The control system plays an important role in the agricultural vehicle guidance system. The previous guidance control system based on PI controller is the only linear, non-dynamic system, and does not work well under the complex cruising condition such as the typical nonlinear, dynamic system in the farm fields. This paper developed an Adaptive Cruise Control (ACC) system based on agricultural vehicle control system for improving its efficiency based on ARM microprocessor and PID algorithm. The real-time experiments on YTO plough tractor achieved better performance index characterizing the operation of the proposed ACC system. This work may provide a proper scheme compared with the present existing linear ACC which can be replaced by a better, nonlinear, more efficient adaptive control system.
\end{abstract}

Keywords: ARM processor, PID algorithm, agricultural vehicle guidance, Adaptive Cruise Control.

\section{INTRODUCTION}

ACC (Adaptive Cruise Control) has been mainly used in aviation and automotive for a long time, but is widely used in the agricultural vehicles because its driving condition is quite complex, dynamical or nonlinear, and different from the air and the road $[1,2]$. A lot of conventional control techniques have been used for ACC which are assumed to be completely known and well understood. In most practical applications, however, the systems to be controlled are dynamical or nonlinear and the fundamental physical processes in it are not completely known as a priori. These system uncertainties are extremely difficult to manage even with the conventional adaptive techniques [3]. All of these special systems require a highly efficient control system for agricultural vehicles. This paper aims at designing an ACC system for agricultural vehicles using PID algorithm and ARM CPU (Central Processing Unit). The procedure of PID described in this paper is part of the development of the ACC system which is an extension of the well-known Cruise Control. In addition to the basic functionality of a Cruise Control, the ACC measures the distance for guiding agricultural vehicles and adjusts the speed in order to keep an appropriate distance. This work is an extension of an earlier approach towards using PID for intelligent vehicle guidance.

\section{ACC ARCHITECTURE DESIGN}

Cruise control is a system that takes charge of controlling the throttle from the driver and cruising the vehicle at a

*Address correspondence to this author at the Medical Instrument Department, Luohe Medical College, Luohe, 462002, China; E-mails: lixingshan@126.com,362764053@qq.com preset constant speed. Cruising control may also maintain string stability in case of multiple vehicles streaming in the fields. Generally, the driver holds the vehicle steady during the drive using the accelerator pedal. Cruise control relieves the driver from that duty and the driver hands over the charge to the ACC when the road conditions are suitable (no strong winds or fog, or there are not wet or icy) and, if the vehicle is cruising at high speed, when there is no heavy traffic, the driver resumes the charge in such conditions.

The block diagram of an ACC embedded system is shown in Fig. (1).

This is a typical embedded system, which is one of the most used systems today in automotive electronics. Its basic functions are as follows. It provides RTC and watchdog timers for the tasks, real-time control for all electronic, electromechanical and mechanical systems; adaptive cruise control (ACC) to maintain constant speed, front switches and display controls port for alarm signals. The CPU S3C2410X carries out malfunction diagnostic analysis through comparing the recorded normal parameters with real time data, and then gives the alarm warning. The warning is issued by displaying pictograms and by raising sound alarms through buzzer. Moreover, it provides some communication ports, such as, SCI (Serial communication Interface), Tx/Rx (Transmitter and Receiver), CAN (Control Area Network Interfaces) and MMI (Multi-Media Interfaces).

The main functions of the ACC system are as following:

\section{(1) The Data Acquisition System (DAS) unit}

This unit determines the current time, data display, updating, recording of instance of malfunctions and periods of normal functioning. Updating can also be done by periodically synchronizing time with time signals from radio broadcasts. 
Cruise control panel

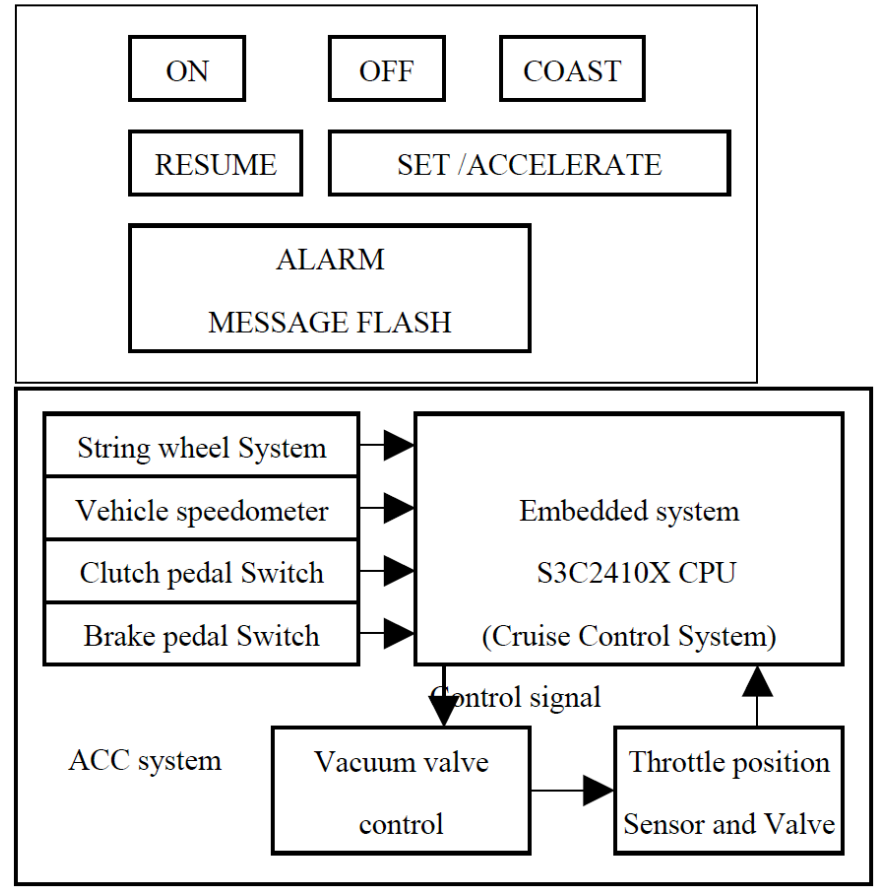

Fig. (1). Block Diagram of An ACC Embedded System Based on ARM.

Moreover, it also determines:

External temperature and internal temperature;

Total distance covered on odometer and for ACC;

Speed of vehicle in $\mathrm{km} / \mathrm{h}$ for the $\mathrm{ACC}$, and speedometer speed warning;

Engine speed in r.p.m.(revolution per minute);

Coolant temperature, periods of its excess above $115^{\circ} \mathrm{C}$ and constancy within $80^{\circ} \mathrm{C}$ to $90^{\circ} \mathrm{C}$, illumination levels at display panels and inside the vehicle;

Fuel level (empty, R1,R2, R3, 1/4, 3/8, 1/2, 3/8, 3/4, 7/8, or Full);

Oil pressure for determining starting oil pressure, warning system activation for engine speed above 5000r.p.m and alarm system activation above 15000r.p.m;

Presently engaged gear information;

Front-end vehicles' distance;

It also sends output to the pedal system for applying emergency brakes and the driver to replace ACC driving vehicle and receiving inputs from the brake switch for brake activities.

(2) For controlling a front-end panel, following steps are followed: The cruise control main switch has seven functions: SET, - (COAST), TAP-DOWN, RES (RESUME), + (ACCEL), TAP-UP, and CANCEL. The SET, TAP-DOWN, and - (COAST) functions, and the RES (RESUME), TAP$\mathrm{UP}$, and + (ACCEL) functions are operated with the same switch. The cruise control main switch is an automatic return type switch which is turned on only when operated in the directions of the arrows and turned off when it is released. The internal contact point of the cruise control main switch is turned on with the switch operation. Following this, the ECM reads the voltage value that has been changed by the switch operation to control SET, - (COAST), RES (RESUME), + (ACCEL), and CANCEL.

(3) The ARM Controller traces throttle position by the stepper motor position sensor, and uses an adaptive algorithm to calculate and send the control signals to the stepper motor at the vacuum valve actuator. The orifice opening of the vacuum valve controls the throttle valve. The valve is electro-pneumatic. Vacuum creation provides the force via the mentioned procedure. The stepper motor with a worm drive attachment to the throttle can also be directly used instead of vacuum actuator and bellows]. Alarms and message flashing unit issue appropriate alarms and messages flashing pictograms.

(4) It also retrieves the front end-vehicle distance information from a radar or U/ VHF (Ultra/ Very High frequency) attachment at the front string wheel. A stepper motor aligns he attachment so that transmitter of radar maintains the line of sight to the front-end vehicle. The radar system maintains stability of the string and provides warnings of emergency situations.

The scheme of an ACC is selected to understand the RTOS needed for applications. The selected RTOS features make a reliable control system in the vehicle electronics system, which used the algorithm and ports which also maintain the strings' stability. 


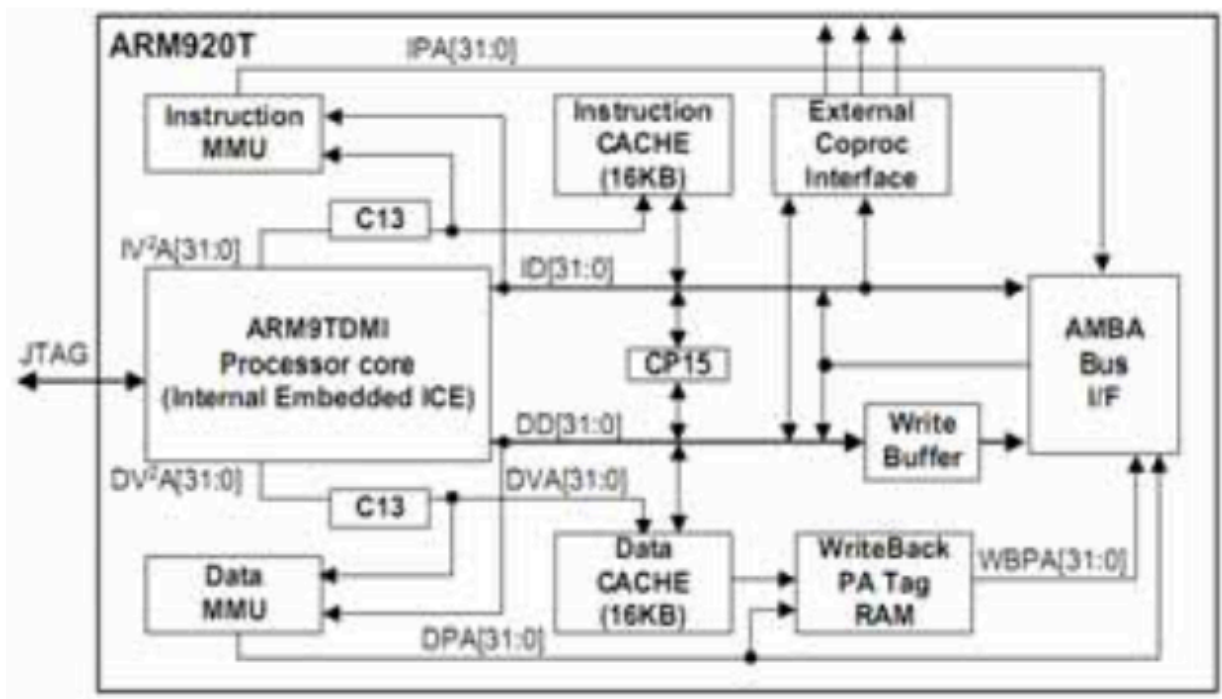

Fig. (2). S3C2410X Block Diagram.

\section{HARDWARE BLOCK DESIGN}

Generally, the vehicle embedded system-based control unit uses a microprocessor, microcontroller, DSP, or ASIP. The device-driver ports and their main functions are as following:

Port Align: It is a stepper motor port. Motor steps up clockwise or anticlockwise on an interrupt signal. The motor is aligned with the radar or UHF/VHF transmitting device in line with the front-end vehicle.

Port Ranging: Time difference timeDiff is read on an interrupt signal received by the port device. A service routine is executed and the radar emits the signal. The sensor receives the reflected signal from the front-end vehicle. The routine disables the interrupts at the beginning and the port device circuit measures the delay between the two instances. The routine enables the interrupts on exiting the critical section. Half of the delay is multiplied by the speed of the task, which measures the speed, and gives the range of the distance of a front-end vehicle.

Port speed: It is a port where, on receiving an interrupt signal, the port device sets a down counter at $\mathrm{N}$ rotation and records the time at this distance. The routine disables the interrupts at the beginning. After the down counter overflows, it again records the time. Moreover, it calculates the difference in time as delta $\mathrm{T}$. The routine enables the interrupts on exiting the critical section. Port Speed is displayed at the speedometer, and the current speed is displayed as Speed-Now, on receiving a signal from Port Rang Rate.

Port Rang Rate: It transmits, a signal, to the port to the Range-Now and Speed-Now and to all other streaming vehicles. It sends signal to the speed port to display Speed-Now on the speedometer.

Port_Brake: Port device applies the brakes or emergency brakes on an interrupt signal. The service routine disables at the beginning and enables the interrupts on exiting the critical section.
It applies the brakes and sends signals to this section and to all the other streaming vehicles. In this study, the Samsung's S3C2410X ARM CPU was used as the controller for its high performances. It is a 16/32-bit RISC microprocessor. This product was designed to provide general applications with low-power, small die size, and cost-effective as well as high-performance micro-controller solution. The S3C2410X includes the separate $16 \mathrm{~KB}$ instruction and $16 \mathrm{~KB}$ Data Cache, System Manager (chip select logic and SDRAM Controller), 3-ch UART, 4-ch DMA, 4-ch Timers with PWM, I/O Ports, RTC, 8-ch 10-bit ADC, IIC-BUS Interface, 2-ch SPI and PLL for clock generation. The S3C2410X was developed using an ARM920T core, 0.18um CMOS standard cells and a memory complier. Its low-power, simple, elegant and fully static design is particularly suitable for efficient controlling of applications. It adopts a new bus architecture called Advanced Microcontroller Bus Architecture (AMBA) [4]. The S3C2410X offers outstanding features with its CPU core, a 16/32-bit ARM920T RISC processor designed by Advanced RISC Machines, Ltd. The block diagram of S3C2410X is shown as Fig. (2).

\section{SOFTWARE DESIGN}

\subsection{Software Scheduling}

An adaptive control means that the algorithm that is used adapts to the current status of the control inputs and does not use a constant set of mathematical parameters in the algorithm equations. Exemplary parameters, which are adapted continuously, include, proportionality constants, integration constants and differentiation constants. Parameters are adapted dynamically. The ACC Model for the adaptive control algorithm in agriculture vehicles is shown in Fig. (3).

Fig. (3) shows how an adaptive control algorithm can adapt and function. It calculates the output values for the control signals [6].

ACC system cycle of actions and task scheduling model are shown in Fig. (4). 


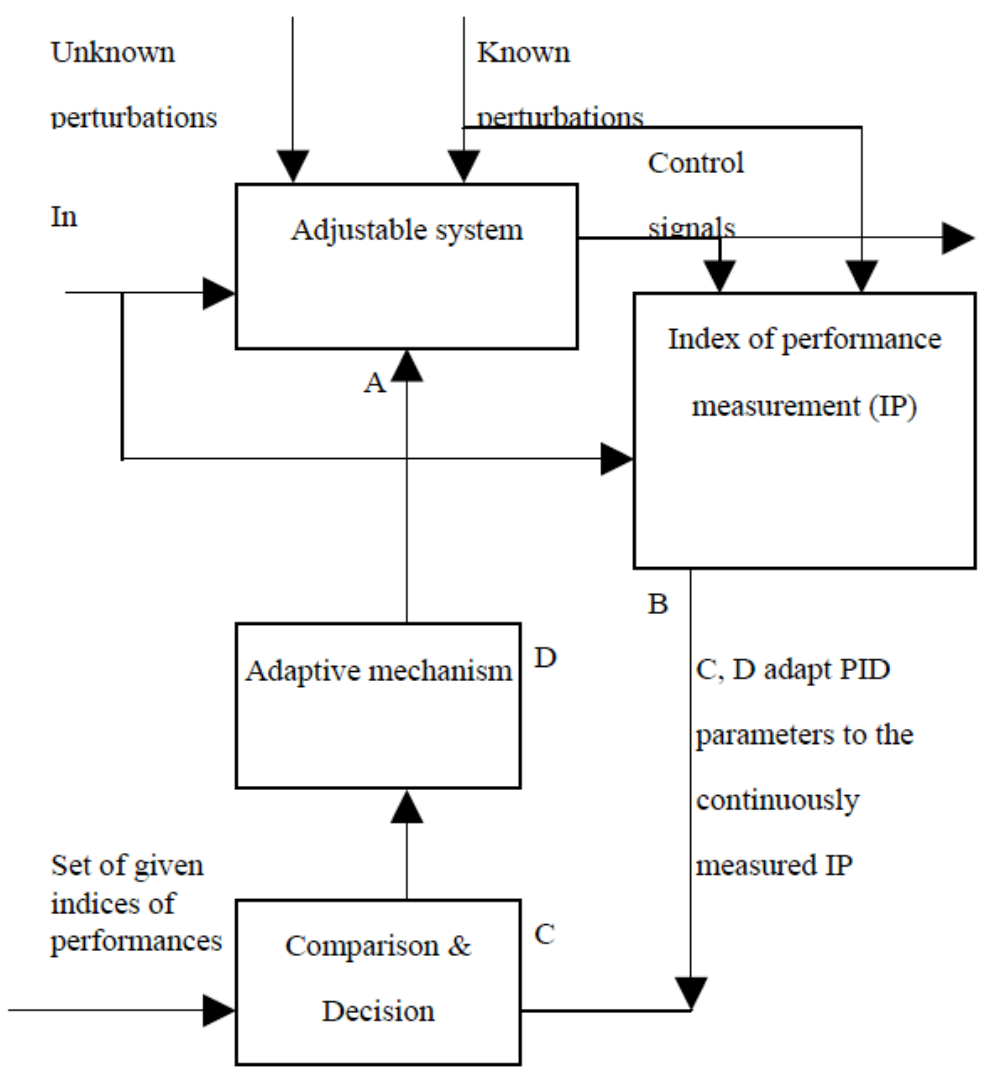

Fig. (3). Model for An Adaptive Control Algorithm Functions.

Task algorithm runs the main adaptive algorithm. It receives inputs from Task Range Rate. The task outputs are provided to the Port Throttle which is attached to the vacuum actuator stepper motor where the cycle ends. After a delay, the cycle starts again from the Task Alignment and receives range and rate error message of other vehicles through Port Rang Rate. It also reads the status of the Port Brake of other streaming vehicles $[5,6]$.

Task Read Range is used for measuring front-end vehicle range. The task disables all interrupts upon entering into the critical section. For real time measurement, Port Ranging is used to find time Diff.

The cycle starts from a task, known as Task Alignment which sends signal to a stepper motor port, Port Alignment and the stepper motor moves by one step clockwise or anti clockwise as directed.

Task Range Rate provides the present range of Task Alignment so that the stepper motor aligns the sensor in line with the front end vehicle. It eliminates the final error by maintaining the string stability of Task Read Range output and by maintaining the vehicle speed from the Task Speed Output. It considers both error values for determining the control system adaptive speed 'Speed-Now', by using the Port Speed which is inversely proportional to the delta $\mathrm{T}$. The ratio $\mathrm{N}$ rotation/ delta $\mathrm{T}$, after applying a filtering function, gives 'speed now'. Port speed is connected to the speed metric system of the DAS, which displays 'speed now' after appropriate filtering function. Port Range Rate also transmits the 'speed now' to other streaming vehicles. Following this, it calculates the range and rate errors and transmits both Range Now and Speed Now through Port Range Rate.

Task Stepper is the task performed to obtain the reading port, known as Port Speed. The Port sends an input as delta $\mathrm{T}$ which represents the time interval between the first and the $N^{\text {th }}$ rotation.

The Task Algorithm generates output for ACC action. Output port generates control signals with a throttle valve. The signals at this port, known as Port Throttle, are calculated as follows. An adjustable algorithm, A, receives inputs of the speed and front object range as well as the unknown and unknown perturbations, $\mathrm{P}$ Unknown and $\mathrm{P}$ Known. It also adjusts the output signal to Port Throttle. In addition, an algorithm, B, eliminates the index of performance IP. While an algorithm $\mathrm{C}$, compares IP with a set of given IP values and algorithm $\mathrm{D}$, as per the adaptive mechanism that adapts the output of $\mathrm{C}$. C, sends the new parameters which are adapted by $\mathrm{A}$.

\subsection{PID Controller Algorithm}

The PID controller algorithm involves three separate constant parameters, and is accordingly sometimes called three-term control; the proportional, the derivative and integral values, denoted by $\mathrm{P}, \mathrm{I}$, and $\mathrm{D}$. These values can be interpreted in terms of time where, D is a prediction of future errors, $\mathrm{P}$ depends on the present error, and I on the accumulation of past errors based on current rate of change [7-9]. The weighted sum of these three parts is used to adjust the process via control element such as the position of a pedal, 


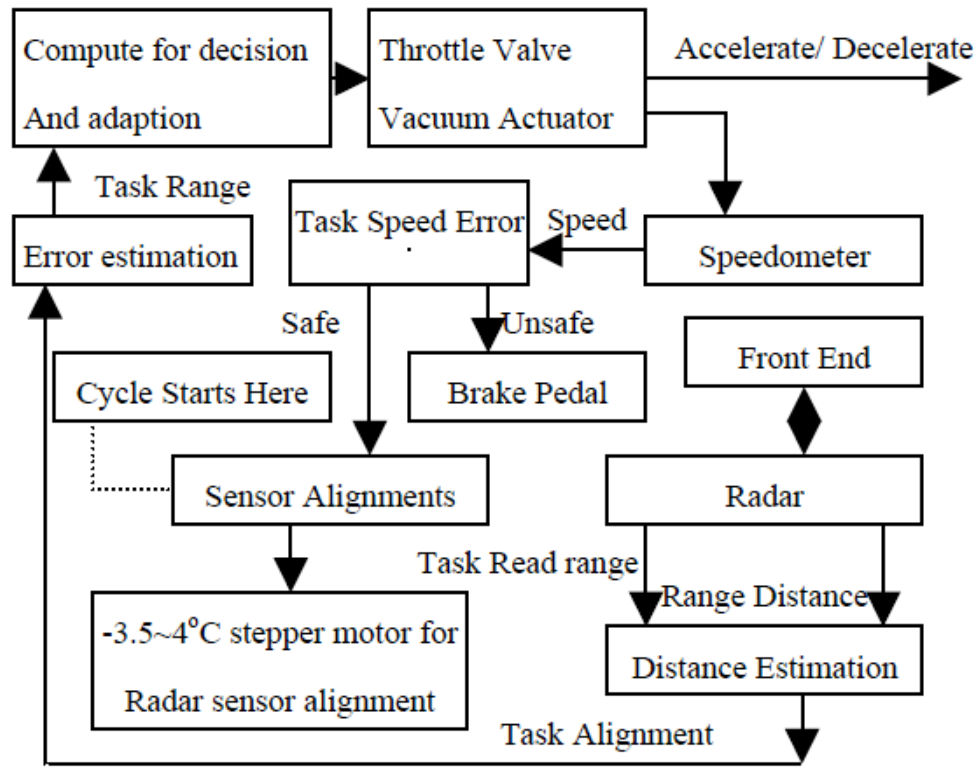

Fig. (4). ACC System Cycle of Actions and Task Scheduling Model.

a control valve, or the power supplied to an engine element. The ACC determines a set point for the throttle and brake, respectively, and provides optical information to the driver by displaying the selected speed and the speed of the proceeding vehicle on the speedometer. In order to make the driver active in the control system, the PID ACC is associated with the active accelerator pedal. The system controls an artificial counterforce at the active accelerator pedal situated at the pedal position which is necessary to maintain the appropriate speed and distance. However, the driver is guided by the controller and is always able to override the actions of the ACC without deactivating the system completely. The driver who receives such alerts in terms of hepatic feedback of the system, is encouraged to use this information to adjust the correct pedal position.

A PID controller has historically been considered to be the best controller in the absence of knowledge of the underlying process. Although using the PID algorithm for control system does not guarantee optimal control of the system or system stability [10]. The controller is used for carrying out control action designed for specific purpose by tuning the three parameters in the PID controller algorithm. The response of the controller can be described in terms of the sensitivity of the controller to an error, the degree to which the controller overshoots the set point, and the degree of system's oscillation.

The PID control scheme is shown in Fig. (5), whose sum constitutes the manipulated variable (MV). The integral, proportional, and derivative terms were added to calculate the output of the PID controller. Defining $u(t)$ as the controller output (Expected steering angle), the final form of the $\mathrm{PID}$ algorithm is as following $[11,12]$ :

$$
\begin{aligned}
& u(t)=M V(t) \\
& =K_{p} e(t)+K_{i} \int_{0}^{t} e(\tau) d \tau+K_{d} \frac{d}{d t} e(t) \\
& e_{i}=S P-P V
\end{aligned}
$$

Where

$K_{p}$ : Proportional gain, a tuning parameter

$K_{i}$ : Integral gain, a tuning parameter

$K_{d}$ : Derivative gain, a tuning parameter

$S P$ : The given value and $\mathrm{PV}$ is the output value.

$t$ : Time or instantaneous time (the present)

$\tau$ : Variable of integration; takes on values from time 0 to the present $t$.

The digital implementation of a PID controller requires the standard form of the PID controller to be discretized in a microcomputer, microcontroller (MCU) or FPGA device [13]. The first-order derivatives were obtained by backward finite differences. The approximated integral term was discretized, with a sampling time $\Delta t$, as follows:

The derivative term is approximated as,

$\frac{d e\left(t_{k}\right)}{d t}=\frac{e\left(t_{k}\right)-e\left(t_{k-1}\right)}{\Delta t}$

Thus, a velocity algorithm for implementation of the discretized PID controller in the ARM MCU was obtained by differentiating $u(t)$, using the numerical definitions of the first and second derivative for solving $u\left(t_{k}\right)$ and finally obtained: 


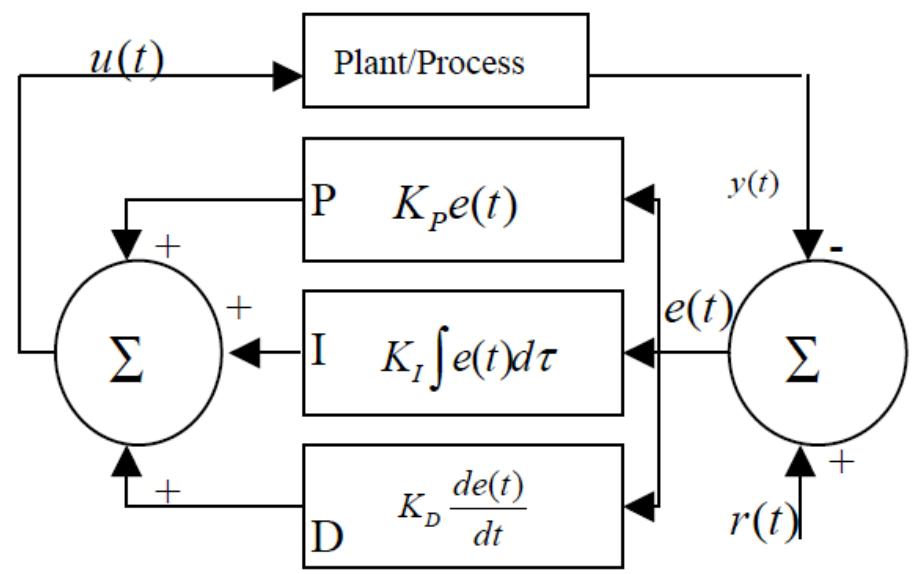

Fig. (5). ACC PID Control Scheme Diagram.

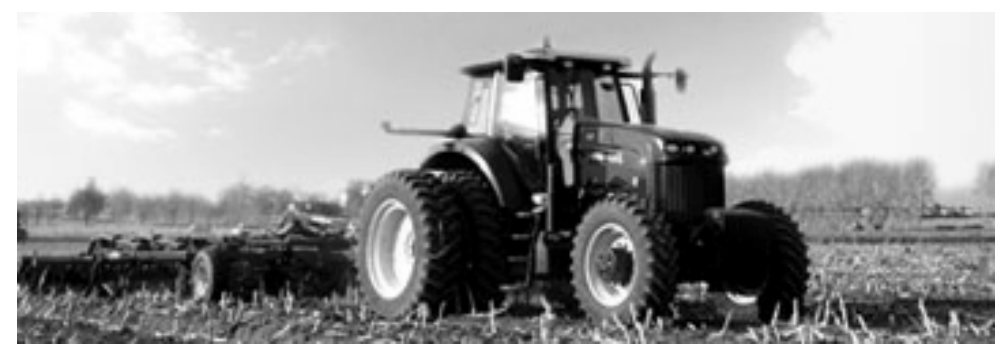

Fig. (6). The Test View of YTO-Plough Tractor.

$$
\begin{array}{r}
u\left(t_{k}\right)=u\left(t_{k-1}\right)+K_{p}\left[\left(1+\frac{\Delta t}{T_{i}}+\frac{T_{d}}{\Delta t}\right) e\left(t_{k}\right)\right. \\
\left.+\left(-1-\frac{2 T_{d}}{\Delta t}\right) e\left(t_{k-1}\right)+\frac{T_{d}}{\Delta t}\right) e\left(t_{k-2}\right) \\
\text { Where, s.t. } T_{i}=\frac{K_{p}}{K_{i}}, T_{d}=\frac{K_{d}}{K_{p}}
\end{array}
$$

Since PID eases search priorities are time-domain tracking and regulation, the corresponding gains are given to assess the frequency-domain properties. Therefore, for higher benchmark plants, the optimized designs were obtained for the twenty order plants as follows [14].

$$
\begin{aligned}
G_{1}(s) & =\frac{1}{(s+1)^{\lambda}}, \lambda=1,2,3,4,8 \\
G_{2}(s) & =\frac{1}{(s+1)(1+\lambda s)\left(1+\lambda^{2} s\right)}, \\
\lambda & =0.1,0.2,0.4 \\
G_{3}(s) & =\frac{1-\lambda s}{(s+1)^{3}}, \lambda=0.1,0.2,0.4,1,2,5 \\
G_{4}(s) & =\frac{1}{(\lambda s+1)^{2}} e^{s}, \\
\lambda & =0.1,0.2,0.4,2,5,10
\end{aligned}
$$

\section{SYSTEM EXPERIMENTS}

When the ARM+PID ACC system was implemented, in order to verify the proposed system, some test drive with YTO-LX2004 for plough action was carried out. YTOLX2004 was produced by the YTO Group Corporation of the Chinese agriculture and construction machinery manufacturer. The result of the test is shown in Fig. (6) and the measured data is represented in Fig. (7).

From Fig. (7), it is obvious that the main target control parameters, such as, target pedal position, acceleration, and velocity were very similar to the actual values. While $K_{p}$ $=1.35, K_{i}=0.075$, and $K_{d}=1.15$, and the system control was optimal.

\section{CONCLUSION}

The ACC system developed based on the ARM CPU combined with the PID controller for guiding the agricultural vehicle had good capabilities. The control algorithm based on the PID compensated the uncertainty and changes in the process dynamics nonlinear system. The PID control algorithm was embedded into the ARM CPU. The practical experiments achieved better performance index characterizing the operation of the control system using the PID controller. The test results showed that the proposed application may be a proper scheme for the already existing linear (PI) control algorithm to be replaced by a better, nonlinear, and more efficient adaptive control system. This work is useful for designing the ACC or likely embedded systems based on ARM and PID. 


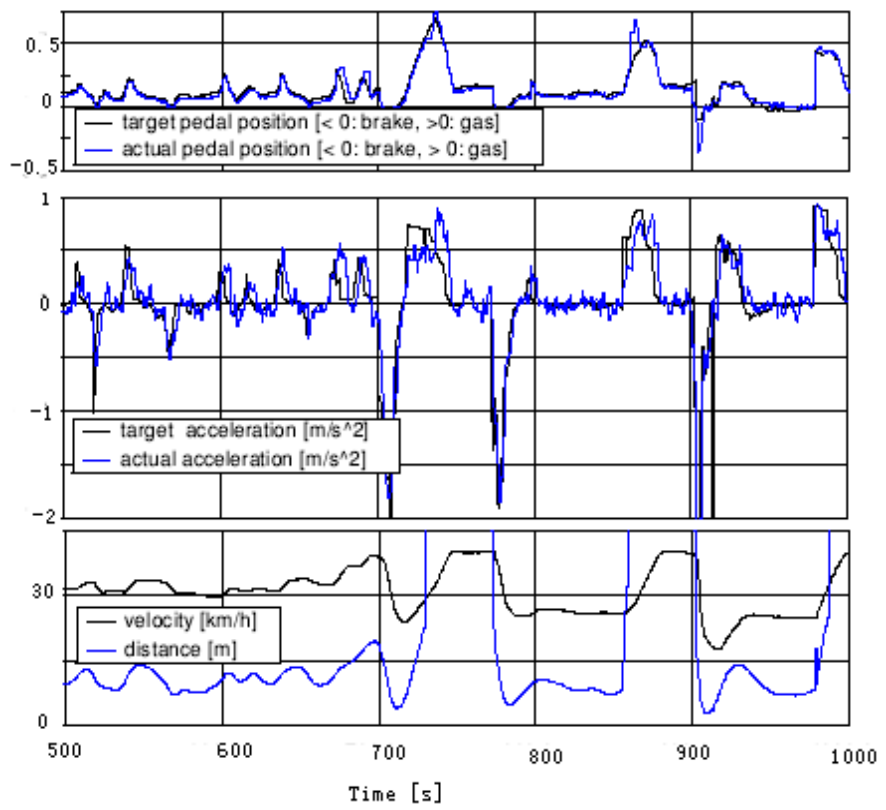

Fig. (7). Test Drive with ARM+PID ACC.

\section{CONFLICT OF INTEREST}

The authors confirm that this article content has no conflicts of interest.

\section{ACKNOWLEDGEMENTS}

This paper is associated with the project of the "NSF of Henna Science and Technology bureau', No. 142300410286. And "the NSF of Henna Education bureau projects", No. 2014B120009.

\section{REFERENCES}

[1] K. Han, Z. Zhu, E. Mao, Z. Song, F. Hu and L. Xu, "Cruise control system of tractor based on automated mechanical transmission", Transactions of the Chinese Society of Agricultural Engineering, vol. 28, no. 4, pp. 21-26, 2012.

[2] D. Xiao, L. Li, P. F. Li, and J. Su, "Modeling and Co-Simulation of Stop and Go Cruise Control System", Advanced Materials Research, vol. 945-949, pp. 1486-1492, 2014.

[3] L. Cai, A.B. Rad, and W. Chan "An Intelligent Longitudinal Controller for Application in Semiautonomous Vehicles", IEEE Transactions on Industrial Electronics, vol. 57, no. 4, pp. 14871497, 2010.

[4] Yue Jun Guo W Q C B. "A New Design of Laser Driver Circuit Controlled by ARM Used in a Gas Detection System Based on TDLAS". Applied Mechanics and Materials. 2014, pp. 21532156.

[5] M. Zhang, X. Lin, Y. Ding, W. Yin, and Y. Qian, "Design of path following controllers based on performance index for agricultural vehicle", Transactions of the Chinese Society of Agricultural Engineering, vol. 28, no. 9, pp. 40-46, 2012.

[6] H. Mousazadeh, "A technical review on navigation systems of agricultural autonomous off-road vehicles", Journal of Terramechanics, vol. 50, no. 3, pp. 211-232, 2013.

[7] A.G. Alexandrov, M.V. Palenov, "Adaptive PID controllers: State of the art and development prospects", Automation and Remote Control, vol. 75, no. 2, pp. 188-199, 2014.

[8] A. Alaimo, V. Artale, C.L.R. Milazzo, A. Ricciardello, "PID Controller Applied to Hexacopter Flight", Journal of Intelligent \& Robotic Systems, vol. 73, no.1, pp. 261-270, 2014.

[9] A.A.T. Maia, J.C. Horta-Gutierrez, R. N.N. Koury, and L. Machado, "Superheating control using an adaptive PID controller", HVAC\&R Research, vol. 2014, no.4, pp. 424-434, 2014.

[10] X. Liang, S. Li, and A.B. Hassan, "A novel PID controller tuning method based on optimization technique", Journal of Central South University of Technology, vol. 17, no. 5, pp. 1036-1042, 2010.

[11] K. Hayashi, and T. Yamamoto "Design of a Data-Oriented Nonlinear PID Control System", IEICE Transactions on Fundamentals of Electronics, Communications and Computer Sciences, vol. E97-A, no. 2, pp. 669-674, 2014.

[12] J. Lee, W. Cho, and T.F. Edgar, "Simple Analytic PID Controller Tuning Rules Revisited", Industrial \& Engineering Chemistry Research, vol. 53, no. 13, pp. 5038-5047, 2014.

[13] John, "Discrete PI and PID Controller Design and Analysis for Digital Implementation". Scribd.com. Retrieved 2011-04-04.

[14] K. J. Åströmand, and T. Hägglund, "Benchmark systems for PID control," In: Proceedings IFAC Workshop, Terrassa, Spain, pp. 165-166, 2000.

(C) Xingshan et al.; Licensee Bentham Open.

This is an open access article licensed under the terms of the Creative Commons Attribution Non-Commercial License (http://creativecommons.org/licenses/by-nc/4.0/) which permits unrestricted, non-commercial use, distribution and reproduction in any medium, provided the work is properly cited. 